\title{
Pyrgi B et la rédaction de la Tabula Cortonensis
}

Koen Wylin

\section{Citer ce document / Cite this document :}

Wylin Koen. Pyrgi B et la rédaction de la Tabula Cortonensis. In: Revue belge de philologie et d'histoire, tome 84, fasc. 1, 2006. Antiquité - Oudheid. pp. 35-44;

doi : https://doi.org/10.3406/rbph.2006.5004

https://www.persee.fr/doc/rbph_0035-0818_2006_num_84_1_5004

Fichier pdf généré le 17/04/2018 


\section{Pyrgi $\mathrm{B}$ et la rédaction de la Tabula Cortonensis}

\section{Koen WYLIN}

En ce qui concerne la deuxième partie du texte de la Tabula Cortonensis $(\S \S \mathrm{V}, \mathrm{VI}, \mathrm{VII})$, il n'existe pas de grandes différences dans l'interprétation des

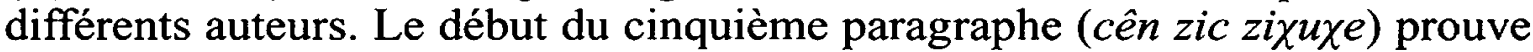
que l'on parle de la rédaction du texte ${ }^{(1)}$. En plus, une liste des témoins présents à cette rédaction ${ }^{(2)}$ est présentée après la formule initiale $\mathrm{cnl} n u \theta e$ malec $^{(3)}$ : $\mathrm{cnl}$ pourrait être l'accusatif pluriel du pronom démonstratif $c a^{(4)}$; male-, qui est à la base de malena (" miroir »), exprime l'idée de voir ou de regarder et il est donc évident que $n u \theta e$ doit avoir une signification plus ou moins comparable ${ }^{(5)}$. Dans la liste des témoins figure le plus haut magistrat, le zila $\theta$ mexl rasnal.

Le dernier paragraphe (VII) commence par une date éponyme (zilci lar $\theta$ al cušus titinal larišalc šalinis aulešla), suivie par une indication topographique (celtinêitišs taršminass) ${ }^{(6)}$, probablement signifiant «au territoire du Lac Trasimène». La séparation des mots peut être faite de plusieurs façons (celtinêi tišs, celti nêitišs, celtinê itišss), mais cela ne changera rien à l'interprétation globale.

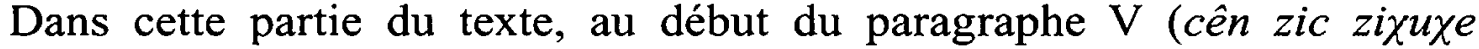
šparzêstis sazleis) la parole-clé est certainement šparza-šparzêstis (sazleis). Le šparza est sans doute un objet particulier, probablement la tablette inscrite, vu que l'objet en question se trouve à un certain endroit, exprimé par une phrase relative qui commence avec in ( $\S$ VII: in $\theta$ uxt cesu) et que le texte même se trouve sur le šparza $(\S \mathrm{V} \text { : šparzête } \theta u i \ldots z i c)^{(7)}$. Dans la même première phrase du cinquième paragraphe, le syntagme šparzêstis sazleis est suivi par in, le

(1) Agostiniani-Nicosia, Tabula Cortonensis, 2000, p.108; Facchetti, Frammenti di diritto privato etrusco, Firenze, 2000, p.80; Maggiani, Dagli archivi dei Cusu. Considerazioni sulla tavola bronzea di Cortona, dans Rivista di archeologia, 25, 2001, p.102; Rix, La seconda metà del nuovo testo di Cortona, dans La Tabula Cortonensis e il suo contesto storico-archeologico. Atti dell 'incontro di studio, (22 giugno 2001), Roma, 2002, p.78.

(2) Comme l'a montré Maggiani (dans Riv.Arch., pp.105, 108), il s'agit de personnes originaires de Cortona.

(3) Agostiniani, Tab.Cort., p.105-107.

(4) Facchetti, Frammenti, p.75, 82.

(5) Facchetti (Frammenti, p.75, 82) traduit: « queste (cose) attestarono e controllarono »; Maggiani (dans Riv.Arch., p.105), par analogie avec male (" (ils) voient »), a traduit nuӨe comme « (ils) écoutent ». p. 105

(6) Agostiniani, Tab.Cort., p.113; Facchetti, Frammenti, p.83-85; Maggiani, dans Riv.Arch.,

(7) Maggiani, Riflessioni sulla Tavola di Cortona, dans La Tabula Cortonensis e il suo contesto storico-archeologico. Atti dell'incontro di studio (22 giugno 2001), Roma, 2002, p.66-67; id., dans Riv.Arch., p.102-103. 
pronom relatif qui caractérise l'antécédent comme non animé(8), une constatation assez bizarre si l'ablatif šparzêstis sazleis était le complément d'agent

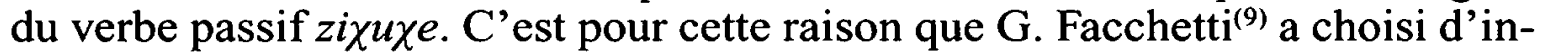
terpréter le šparza sazle comme un collège de magistrats, responsables de la transcription du texte. $\mathrm{H}$. Rix ${ }^{(10)}$ par contre a proposé une autre solution: sazleis serait comme substantif le complément d'agent du verbe et serait qualifié par šparzêstis, analysé à son tour comme šparza-s (génitif) avec l'ablatif du pronom démonstratif ita-is, avec métathèse de la jota (cfr. Өapnests dans le LL). Le résultat pourrait signifier «par le sazle, celui de la tablette». Mais alors surgit une autre difficulté: si la phrase relative in $\theta$ uxt cušu $\theta$ uras šu $\theta i u$ ame dépendait de šparzêstis, on aurait eu l'ordre inverse des mots (sazleis šparzêstis); si elle dépendait de sazleis, il faudrait conclure que in peut aussi être utilisé pour des êtres animés, puisque alors sazle devient le complément d'agent (une

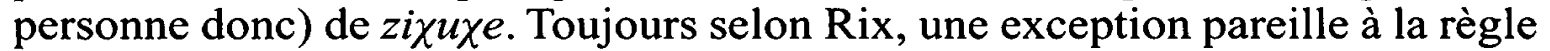
des pronoms relatifs ${ }^{(11)}$ se trouverait dans le LL III/IV, deux colonnes pour le flere in crapsti, ou dans AV 4.1 (aiseras in ecs mene mla $\theta$ ce). Rien par contre ne nous assure que flere (une force numineuse) est considéré comme animé et on peut même discuter l'autre exemple. Voyons donc si d'autres solutions sont possibles.

Maggiani ${ }^{(12)}$ considère šparzêstis comme un locatif avec un élément enclitique, redéterminé à l'ablatif: šparzêt $i<\breve{s} p a r z a-i-t i>\breve{s p a r z e ̂}-s-t i-s$. La valeur de cette construction serait celle d'un locatif instrumental, donc signifiant «ce texte a été écrit sur le šparza sazle». De cette façon sazle indiquerait le matériel de la tablette (bois, bronze,...?). Par contre il faut dire que l'existence d'un tel ablatif du locatif n'a jamais été prouvée; il n'y a donc pas de preuves morphosyntaxiques.

Récemment une autre solution a été proposée par Adiego ${ }^{(13)}$, qui a interprété l'ablatif šparzêstis non comme complément d'agent mais comme ablatif de séparation $^{(14)}$. Cette thèse pourrait être corroborée par des textes parallèles en latin (p.ex. descriptum et recognitum ex tabula aenea). La traduction de la séquence

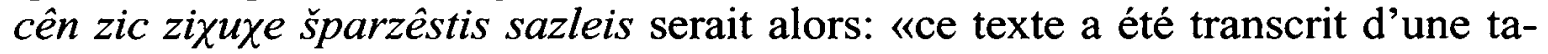
blette sazle (= en bronze, en bois?)».

Seulement, selon moi, il faut toujours analyser exactement la forme šparzêstis. G. Facchetti ${ }^{(15)}$ avait choisi l'interprétation de šparzê-s-ti-s comme ablatif d'un

(8) Agostiniani, Tab.Cort., p.100.

(9) Frammenti, p.80.

(10) dans La Tabula Cortonensis. Atti, p.81-83.

(11) Voir note 8.

(12) dans La Tabula Cortonensis. Atti, p.66-67; id., dans Riv.Arch., pp.102-103.

(13) Ignasi-Xavier Adiego, Two conferences held in March 2004 at the University of California-Los Angeles and at the Workshop on Italic Languages and Dialects, University of California, Berkeley, and titled The Etruscan Tabula Cortonensis: a Tale of Two Tablets? Je n'ai pas pu consulter l'article moi-même; je le prends de l'article de Facchetti (Some New Remarks on the Tabula Cortonensis), présenté en Pologne à l'université de Lodz, à l'occasion d'une conférence "Europe Through Millennia - Languages, Races, Cultures, Beliefs" (Lodz, juin 25-26, 2004).

(14) Pour un tel usage de l'ablatif, cfr. Facchetti, Appunti, p. 39-44.

(15) Appunti di morfologia etrusca, Firenze, 2002, p.83-84. 
substantif avec un élément enclitique šparza-ti. L'analyse de Rix (šparza avec le pronom démonstratif ita, mis à l'ablatif (ita-is)) donnerait le résultat - $t s$, comme on peut l'observer dans êliun-ts au début de l'inscription. Pour Facchetti l'élément -ti(s) a donc une signification inconnue, mais quand même différente de l'élément locatif $-t i$, et de l'élément adjectival $-t i$ (p.ex. paxanati). Contrairement à Facchetti, je me demande si l'élément - $t i$ n'est quand même pas celui des adjectifs. Alors l'adjectif šparza-ti (à l'ablatif) dépendrait du substantif sazleis, qui pourrait signifier «original». La première phrase du paragraphe $\mathrm{V}$ se laisserait alors traduire comme: «ce texte a été transcrit d'un original de tablette, qui ...».

Puis on a la phrase relative «qui a été posé dans la maison des Cušu» (in $\theta$ u $\chi$ ti cušu $\theta$ uras šu $\theta i u$ ame), se référant donc à la tablette originale. Maggiani(16)

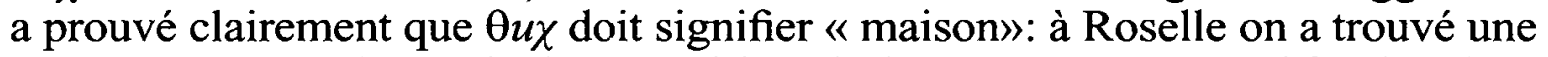
coupe (3ième - 2ième siècle) avec l'inscription [---]-trus apanal $\theta u c l$, qui ne peut signifier que: «moi, don de [---]-tru et de la maison paternelle». Il nous paraît donc évident que le texte original du contrat se trouvait chez les frères Cušu. Ce sont eux qui ont donné à Petru le droit d'utiliser certains terrains, décrits au premier paragraphe ${ }^{(17)}$.

La phrase suivante est assez problématique: tal šu $\theta$ ivenas ratm $\theta u \chi t$ cesu tltel têi.

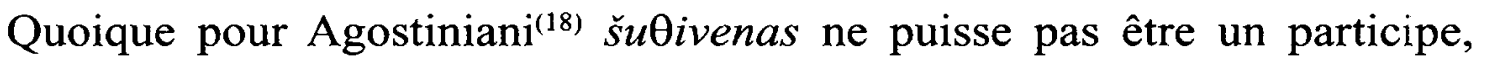
puisque la forme serait, selon lui, construite sur le participe $\breve{s} u \theta i u$, la plupart des auteurs actuels semblent d'accord pour accepter l'hypothèse participiale. En effet, la forme šu $\theta i u$ n'est pas (nécessairement) un participe, mais doit plutôt être considérée comme une forme perfective, mais non spécifiée sur le plan syntaxique ${ }^{(19)}$. Selon Rix, il faut considérer la forme šu $u$ ivenas comme passive («essendo depositato») (20), puisqu'il s'agirait du texte même dont on a parlé dans la phrase précédente et donc, toujours selon Rix, c'est le texte qui a été déposé.

Plus proche de la vérité me paraît Maggiani(21): vu que l'original du texte

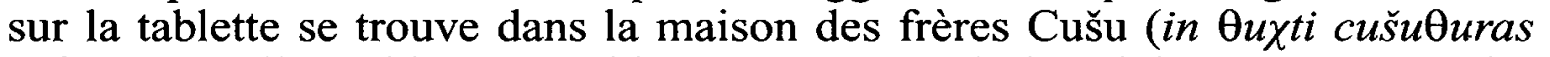
$\check{s} u \theta i u$ ame), il semble acceptable qu'une transcription doit se trouver également chez Petru. On ne peut pas exclure que la phrase tal šu $\theta$ ivenas ratm $\theta u \chi t$ cesu tltel têi concerne cette idée. Mais il n'est pas prouvé qu'il faut analyser $\check{s} u \theta i v e n a s$ comme un adjectif substantivé («qui appartient à qui dépose $=$ objet déposé»), comme le veut Maggiani.

(16) dans La Tabula Cortonsis. Atti, p.67; id., dans Riv.Arch., p.103-104.

(17) Wylin, The first chapter of the Cortona inscription in Etruscan News, winter 2006, p. 6-7 à consulter sur www.nyu.edu/fas/center/ancientstudies.

(18) Tab.Cort., p.111.

(19) Wylin, Il verbo etrusco, Roma, 2000, §§ 8, 15.2.

(20) dans La Tabula Cortonsis. Atti, p.83.

(21) dans Riv.Arch., p.106. 
A la lettre $\breve{s} u \theta i v e n a s$ est un participe (-as) médial $(-\mathrm{n})$ parfait $(-u)^{(22)}$, signifiant donc «s'étant déposé». Il n'est pas impossible qu'il s'agisse ici de la présentation du texte, exprimée en latin avec le verbe ponere, qui peut signifier «déposer en écrit». Ce participe pourrait être utilisé substantivement («une déposition écrite»). Dans ce cas tal peut bien être le génitif de $t a$, même s'il est vraisemblable que dans d'autres textes les formes pronominales avec $-l$ sont des pluriels ${ }^{(23)}$. En plus un nominatif pluriel serait quasi impossible, car il serait le sujet de quel verbe au juste? Et je crois avoir prouvé qu'il existe des textes ou $s a l$ doit être le génitif d'un pronom $s a^{(24)}$.

La séquence tal $\breve{s} u \theta$ ivenas ratm $\theta u \chi t$ cesu pourrait donc être traduite comme: «de cet (original) une déposition écrite a été posée dans la maison». Que ratm soit en effet un adverbe, comme généralement admis ${ }^{(25)}$, n'est pas tout à fait certain. Maggiani ${ }^{(26)}$ a proposé de traduire ratm comme «exemplaire/copie», évidemment basé sur sa traduction de šu $\theta$ ivenas («de cette chose déposée une copie a été posée»). Si par contre šu resterait un adverbe, mais signifiant peut-être simplement «aussi») (rat- $(u) m \sim$ quo-que! $)^{(27)}$. On aurait donc: «de ce (texte) une copie a été posée aussi dans la maison...».

Une conclusion pourrait être que tltel têi est une référence à la seconde partie du contrat, c.à.d. Petru et sa femme: têi peut être le locatif de $t a$, renvoyant à $\theta u \chi t$; tltel alors sera un complément de têi, probablement un génitif du locatif. Donc on a: «dans la maison, dans celle de celui-là) ${ }^{(28)}$, donc dans la maison de Petru, qui, ne l'oublions pas, a été mentionné dans la phrase précédente. Je crois donc que la séquence $\theta u \chi t i$ cušu $\theta$ uras šu $\theta i u$ ame se trouve parallèle à $\theta u \chi t$ cesu tltel têi. Il est donc probable que le pronom démonstratif ta s'utilise ici au sens du latin hic-ille (le dernier - le premier).

Puisqu'on a déjà parlé de šparza et de ratm, il nous reste peu de choses à dire de la section VII, que j'ai déjà traitée dans un autre article concernant

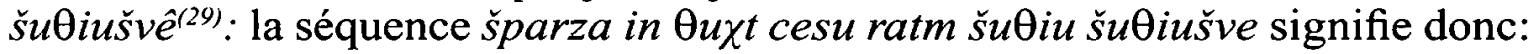
«la tablette qui a été déposée dans la maison, est également/aussi (?) déposée dans les résidences (šu

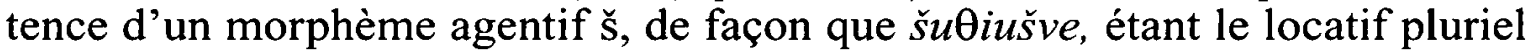
de * $̌ u \theta i u-\check{s}$, puisse signifier «résidence» («quelque chose qui fait demeurer, qui pose les gens à l'intérieur»). $\theta u \chi$ en effet pourrait être un édifice public, vu

(22) Wylin, Forme verbali nella Tabula Cortonensis, dans Studi Etruschi, 65-68, 2002, p.221.

(23) Facch, Appunti, p.26, 28-34.

(24) Wylin, Un terzo pronomelaggettivo dimostrativo etrusco "sa", dans Studi Etruschi, 70, 2004, p. 214-220.

(25) Agostiniani, Tab.Cort., p.110 et Rix, dans La Tabula Cortonsis. Atti, p.80 («rituellement»); Fachetti, Frammenti, p.79 («précisément»).

(26) dans Riv.Arch., p.105.

(27) Il faut admettre que les autres séquences avec ratm (LL 10.4, 20 et TC 27) sont difficiles à interpréter, mais n'excluent pas la traduction «aussi».

(28) Maggiani, dans La Tabula Cortonsis. Atti, p.66.

(29) Wylin, Un morfema agentivo etrusco, dans Archivio glottologico italiano, 89, 2004, 1, p.111-127. 
que le mot n'est plus spécifié concrètement ${ }^{(30)}$. En plus le paragraphe commence par une date éponyme, donc avec «maison» on pouvait bien indiquer la résidence des zila $\theta$. On voudrait insister ici que d'un autre texte important et officiel, celui de Pyrgi A, il est probable qu'il existait deux versions, l'une conservée auprès du temple (munistas $\theta$ uvas tameres), la seconde dans la résidence du zila $\theta$ (atranes zilacal) ${ }^{(31)}$. Une autre possibilité me semble le fait que l'on renvoie de nouveau à la tablette originale dans la maison des frères Cušu; de cette tablette on conserve une copie, non seulement dans la maison de Petru, mais également dans la résidence de quatre personnes différentes.

Etudions maintenant la séquence assez longue šians šparzête $\theta$ ui šalt zic fratuce cušu $\theta$ uras larišališvla pêtrusc šcêvas pěss tarðianês. La structure de la première partie est évidente: šians au nominatif doit être le sujet; šparzête $\theta u i$ šalt semble un complément locatif; zic, vu la position devant le verbe fratuce, est sans doute l'objet direct du verbe ${ }^{(32)}$.

Interpréter šians dans le sens de "père»" ${ }^{(33)}$ est très précaire. Facchetti ${ }^{(34)}$ croit qu'il s'agit d'un fonctionnaire quelconque, quelqu'un qui est responsable de l'action fratuce, qui ne peut signifier que «inciser, graver», puisque l'action se déroule sur la tablette (šparzête) ${ }^{(35)}$. Il faut à mon avis retenir l'avis de Facchetti ${ }^{(36)}$ : šians pourrait bien être lié à sanus, dans le sens de «intègre, sage, prudent, prospère», utilisé ici comme adjectif substantivé et applicable à flere (Pe 3.3) de même qu'à un fonctionnaire. Donc «le šians a incisé le texte ici sur la tablette»».

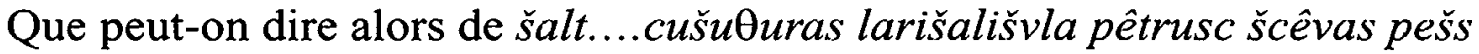
tarxianês que je crois être un seul syntagme? Commençons par les deux derniers mots: peš semble une propriété, une interprétation basée sur l'indication pešc špante au premier paragraphe, indiquant que le peš doit être quelque chose qui peut se trouver dans la plaine ${ }^{(37)}$. C'était Maggiani ${ }^{(38)}$ qui a proposé l'interprétation fundus, se basant sur les quatre séquences dans lesquelles figure le $\operatorname{mot}^{(39)}$. Personne ne discute le fait que pešs tarzianês est un ablatif ${ }^{(40)}$. Je n'ajoute donc aucune foi à la théorie de Facchetti qu'il s'agirait d'une référence au mythique Tarchie. Il y a la combinaison avec fundus et en plus, une telle référence dans un texte comme celui de la tablette me semble peu

(30) A cet égard, je suis donc d'accord avec Maggiani, dans Riv.Arch., p.105.

(31) Wylin, Esiste una seconda lamina A di Pyrgi?, dans Parola del Passato, 328, 2003, p. 61-65.

(32) Maggiani, dans Riv.Arch., p.104.

(33) cfr. Colonna, La dea Cel, dans Rivista di storia antica, 6-7, 1976-1977, p.58-59; id., Note di lessico etrusco, dans $S E, 48,1980$, p.167; id., A proposito degli dèi del Fegato di Piacenza, dans $S E, 59,1993$, p.134-136.

(34) Frammenti, note 465.

(35) Rix, dans La Tabula Cortonensis. Atti , p.84.

(36) Appunti, p.22-23.

(37) Déjà Agostiniani, Tab.Cort., p.102.

(38) Dans La Tabula Cortonsis. Atti, p.72.

(39) Voir aussi Wylin, The first chapter of the Cortona inscription, dans Etruscan News, 2006, p. 6-7.

(40) Maggiani, dans Riv.Arch., p.104; id., dans La Tabula Cortonsis. Atti, p.72; Facchetti, Frammenti, p.69; Note etrusche, dans Archivio glottologico italiano, 88, 2003, 2, p.207-208. 
probable. Acceptons donc l'interprétation de Maggiani qu'il s'agit d'un ablatif indiquant la provenance de Petru. Comme a observé Maggiani, tarxi est un nom assez fréquent dans la région de Sienne.

On peut en conclure que šalt appartient aux frères Cušu et à Petru Šceva, provenant du fundus Tarcianus. L'interprétation dépend donc de šalt, et je ne crois pas qu'il s'agisse d'un locatif du pronom $-\check{s} a^{(4 t)}$, qui est, au moins dans tous les textes connus à présent, toujours enclitique. Et il faut bien tenir distinct le pronom enclitique $-\check{s} a$ d'un autre pronom $s a$, dont j'ai prouvé une signification comparable à celle du grec $\alpha$ ùंó ${ }^{42)}$.

En ce qui concerne $\check{s} a$, je suis persuadé avec Facchetti ${ }^{(43)}$ qu'il faut tenir compte de šelace dans Pyrgi B (Cr 4.5): nac Oefarie veliiunas / Oamuce cleva

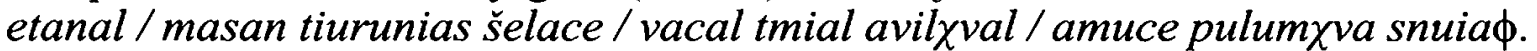
Il me semble qu'on ne peut pas discuter le fait que trois formes verbales figurent dans l'inscription: Өamuce, šelace, amuce. Puisque $\theta$ amuce signifie apparemment "construire» (cfr. Ta 5.2, $\mathrm{Cr} 4.4$ ), le verbe a l'aspect d'un verbe bivalent. Oefarie veliiunas doit être le sujet; il en résulte que cleva (etanal) pourrait fonctionner comme objet ${ }^{(44)}$. Déjà selon Pallottino ${ }^{(45)}$ et Pfiffig ${ }^{(46)}$ cleva devait être l'objet, comme il indique un sacrifice dans la Tabula Capuana. Pallottino considérait etanal comme un génitif du pronom démonstratif, Pfiffig comme un génitif d'un nom. Dans Etruskische Bauinschriften ${ }^{(47)}$, Pfiffig a interprété cleva comme un autel et Etanai serait une épithète de Uni. Par conséquent šelace devrait signifier «consacrer». Olzscha ${ }^{(48)}$ a également traduit la séquence $\theta$ amuce cleva dans le sens de: «a construit un cleva». Et récemment Steinbauer ${ }^{(49)}$ a interprété à son tour cleva comme l'objet du verbe et probablement l'objet de $\theta$ amuce.

Devoto $^{(50)}$ par contre a lié cleva au verbe suivant šelace en traduisant «a sacrifié un cleva». Dans le même sens Facchetti ${ }^{(51)}$ croit que l'objet de $\theta$ amuce est omis, de façon que cleva devient l'objet de šelace.

Cependant l'argument de la bivalence de $\theta$ amuce me semble assez fort et en plus il y a l'analogie possible entre cleva et cluvenias - clivinia $^{(52)}$. Comme j'ai expliqué dans mon article, je crois bien que $s a$ peut être considéré comme un

(41) Cfr. Adiego, (̌salt <*(i)šala- $\theta i$ : Two conferences held in March 2004); Facchetti (Some New Remarks on the Tabula Cortonensis) par contre a proposé de deriver šalt de * (i)šale- $\theta$ i/te (cfr. *(i)cale- $\theta i / t e$, dans Appunti, p. 27, 56, 76-77). En tous cas, si šalt est le locatif d'un pronom, il figure quand même trop de locatifs dans l'interpretation de cette séquence: «En ce qui concerne ces choses (tltel), sur ceci (têi), le šians, sur la tablette (šparzête) ici ( $\theta u i)$, sur

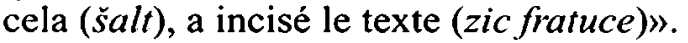

(42) Cfr. Wylin, Un terzo pronome/aggettivo dimostrativo etrusco "sa", dans SE, 70.

(43) Appunti, p.72.

(44) Schulze-Thulin (Zur Worstellung im Etruskischen, dans $S E, 58,1992$, p. 188) y reconnait une construction SVO: Өefarie veliiunas $\theta$ amuce cleva etanal.

(45) Scavi nel santuario di Pyrgi, dans Archeologia Classica, 16, 1964, p.100-101.

(46) Uni-Hera-Astarte (Österreichische Akademie der Wissenschaften. Philosophischhistorische Klasse. Denkschriften, 88), Wien, 1965, p.36.

(47) 1972, p.29.

(48) Die punisch-etruskischen Inschriften von Pyrgi, dans Glotta, 44, 1967, p.97-98.

(49) Neues Handbuch des Etruskischen, 1999, p.207-8.

(50) Considerazioni sulle lamine auree di Pyrgi, dans SE, 34, 1966, p.215.

(51) Appunti, pp.24, 70.

(52) Cfr. Wylin, dans $S E, 70$, p. 224. 
pronom comparable à $\alpha$ $\tau o ́ c ̧$ Dans cette ligne d'idées la séquence sal cluvenias turuce de Pyrgi A pourrait signifier que Thefarie Velianas a donné le tmia et les heramašva, qu'il avait fait construire (Өemiasa), à Uni, suite à une demande de la déesse même. Autrement la mention de cette demande serait la seule chose du texte phénicien qui ne figure pas dans la version étrusque. La séquence sal cluvenias me semble donc un génitif dédicatoire. Facchetti m'a fait savoir que selon lui le génitif d'un pronom $s a$ dans un texte archaïque comme celui de Pyrgi devrait être *sala. Il faut admettre quand même que Pyrgi A, tout en étant archaïque (de la phase finale), présente déjà la contraction tameresca (et non *tameraisca). En plus, dans d'autres textes archaïques on rencontre les génitifs ipal $(\mathrm{Cr} 4.10)$ et ital $(\mathrm{TC})^{(53)}$.

Si en effet cluvenias est dérivé de cleva ${ }^{(54)}$, il faudra considérer cleva comme un substantif, indiquant un vœu. Ensuite on peut se demander si etanal est un génitif se référant à cleva ( «un vœu de l'etana») ou plutôt un accusatif pluriel archaïque du pronom ta, comme l'est la forme récente $c n l$ vis-à-vis de $c a$ dans la Tabula Cortonensis. De cette façon etanal pourrait être utilisé comme adjectif, déterminant $\operatorname{cleva}^{(55)}$ : «Thefarie Veliiunas a construit ces vœux (c.-à-d. le tmia et les heramašva)».

On peut donc conclure que nac $\theta$ efarie veliiunas $\theta$ amuce cleva etanal forme la première phrase, suivie à son tour de masan tiurunias šelace. Déterminer si

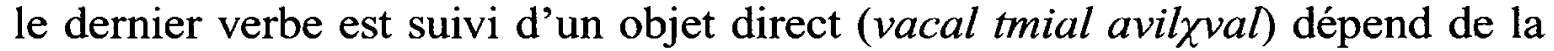
signification et de la valence de šelace.

Alors que pourrait signifier masan? La plupart des commentaires de l'inscription ont interprété ce mot comme l'indication d'un mois (en combinaison avec tiuriunias); une telle interprétation est sans doute basée sur LL 12.10 ( $\theta$ unem

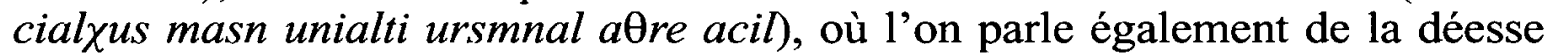
Uni (cfr. tiuri-uni-as) et ou masn figure après un numéral ${ }^{(56)}$. Pour Facchetti(57) par contre masn exprimerait la répétition, ce qui s'adapterait bien à tiuriunias, interprété comme un rite mensuel.

Quoique le terme masnur soit assez incertain (LL 10.12), il faut quand même en tenir compte, puisqu'il peut s'agir d'un pluriel animé, de façon que masn doive être une certaine personne, p.ex. dans une fonction sacerdotale. Ce qui, selon moi, s'adapterait bien aux deux passages du Liber Linteus. Il y a d'abord LL12.9-12: la séquence $\theta$ unem cialxus masn unialti ursmnal a $\theta$ re acil pourrait signifier «le 29 dans le temple de Uni Ursmna il est nécessaire ${ }^{(58)}$ que le masn

(53) Facchetti, Appunti, p.30. Une autre possibilité de laquelle je n'ai pas tenu compte dans mon article est que sal pourrait être le numéral «deux» (cfr. $e$-sl). Même dans ce cas la signification de cluvenias peut se maintenir: «Th.V. a donné les deux (c.-̀̀-d. tmia et heramašva), suite à la demande (de la déesse)».

(54) Steinbauer, (NHE, p.201) n'exclut pas la possibilité d'une erreur pour *clevunias.

(55) On pourrait penser à une règle semblable à celle d'Agostiniani en ce qui concerne les numéraux, càd que peut-être les substantifs inanimés n'exprimaient pas le pluriel quand le nombre était déjà indiqué par un autre mot, par exemple par un pronom.

(56) Pallottino, dans $A C, 16,1964$, pp.101-102; Olzscha, Die punisch-etruskischen Inschriflen, dans Glotla, 44, 1967, p.99.

(57) Appunti, p.71.

(58) En ce qui concerne la formule acil (ame), voir déjà Olzscha, Etruskisch «acil», in $S E, 29,1961$, p.158 sqq. 
fasse l'action $a \theta r$-»). L'autre séquence LL.7.12-14 (cntnam $\theta$ esan masn zelv(e) $\theta$ murss etnam $\theta$ acac ušli nex še acil ame) a une structure un peu parallèle: comme dans LL 12.9-12 masn se trouve entre une indication temporelle ( $\theta$ unem cialxus - Өesan) et un locatif (unialti - zelve $\theta$ ). Donc également ici il est bien possible que le masn doive faire l'action nexš-. Et en plus on ne peut pas oublier que l'on se trouve dans un calendrier religieux exprimant des actes d'offrandes, qui doivent quand même être accomplis par un prêtre quelconque.

Dans Pyrgi B il ne me semble donc pas impossible que la masan fasse l'action, exprimée par le verbe šelace. Dans ce cas tiuriunias doit être soit un génitif de dédicace, soit un complément de masan. On pourrait se demander s'il s'agit d'une indication temporelle (dans le sens de rite mensuel?) ou plutôt d'un rite lunaire (tiu, tivr). Dans le cas de LL 7.12-14 le masn doit faire quelque chose au lever de soleil $(\theta e s a n)$ et pendant le jour $(u s ̌ l i)^{(59)}$. On pourrait s'imaginer qu'ici une certaine action doive s'effectuer à la pleine/nouvelle lune.

Il me semble justifié de considérer vacal comme l'objet direct de šelace. Comme je l'ai dit ailleurs ${ }^{(60)}$, il est plus logique et acceptable dans tous les contextes de traduire vacl dans le sens de «offrande». En général les génitifs tmial avilxval sont considérés comme une unique séquence, traduite comme «du temple des années $\rangle^{(61)}$. Par contre il faut tenir compte de l'interprétation de Giannecchini ${ }^{(62)}$ que snuia $\varphi$ pourrait être un numéral, indiquant peut-être douze ${ }^{(63)}$. Il y a plusieurs éléments qui pointent dans cette direction: dans un certain nombre de langues onze et douze ne sont pas des numéraux composés; les numéraux ont souvent des structures internes parallèles (cfr. sem- $\phi$, nur- $\phi$ ); dans LL 6.4 snuia $\phi$ se trouve après pe $\theta$ ereni, où l'on trouve ailleurs également des numéraux $(10.2,11.8)$; dans LL 6.1 il y a la séquence aceva snuiu $\phi$, tout à fait parallèle à pulum $\chi v a$ snuia $\varphi$ de Pyrgi B. Finalement on pourrait mentionner le fait, même si cela ne prouve rien, que l'on a trouvé auprès de la tablette 12 clous (auprès de la tablette A il y en avait seulement 10), ce qui pourrait se référer aux clous annuaires. Dans ce cas on pourrait accorder avec Giannecchini que les pulum $\chi v a$ snuia $\varphi$ pourraient être les pulum $\chi v a$ avil $\chi v a l$. J'estime donc bien acceptable qu'il faut séparer avilðval de vacal tmial, de façon que la dernière séquence de l'ins-

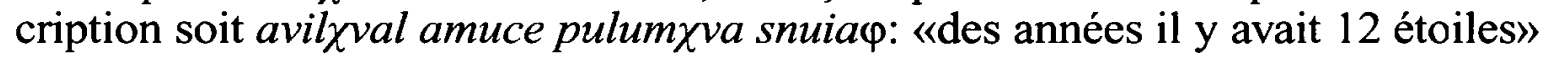
c.-à-d. que 12 années étaient passées depuis la donation du temple.

(59) Facchetti, Appunti, p.71.

(60) $V E$, pp.123, 188. Je ne suis pas d'accord non plus avec l'interprétation «louange», comme supposé par Facchetti (Appunti, pp.102-106). L'auteur ne donne pas de preuve pour cette interprétation; en plus dans LL 8.16-17 (日ezin fler vacl etnam...) il est obligé de supposer un impératif omis: «fa (= sacrifica) la vittima ed (esegui) pure la lode». Dans LL 5.16 Facchetti ne mentionne pas que vacl $\theta$ esnin est probablement l'objet direct de celi šu $\theta$ («dépose sur la terre»). Il me semble assez difficile de déposer une louange sur la terre. Et il faut remarquer aussi que dans le Liber Linteus la racine hec s'utilise avec des offrandes concrètes, tel le vin (vinum dans LL $4.9,4.14,9.6$ ), et dans LL 11.4 on trouve la séquence vacl hexz.

(61) Pallottino, dans $A C, 16,1964$, p.103; Facchetti, Appunti, p.70.

(62) Un'ipotesi su 12, dans Parola del Passato, 52, 1997, 3, p.190-204.

(63) Facchetti (Appunti, p.70) a interprété d'une manière très hypothétique la séquence comme une exclamation «100 étoiles!». 
Passons maintenant à šelace. Dans un contexte comme décrit plus haut, que pourrait signifier ce verbe, tout en tenant compte de šal dans la Tabula Cortonensis? Voici de nouveau les deux séquences:

- masan tiurunias šelace vacal tmial

(«le masan tiurunias a fait l'action šal l'offrande du temple»)

- šians šparzête $\theta u i$ šalt zic fratuce cušuӨuras larišališvla pêtrusc šcêvas

(«le fonctionnaire sur la tablette ici šalt a inscrit le texte des Cušu et de Petru Šceva»)

Auparavant šelace a toujours été traduit comme un verbe d'offrande ${ }^{(64)}$. Facchetti $^{(65)}$, sans pouvoir le prouver, a opté pour une interprétation dans le sens d'une action rituelle de la part d'un prêtre vis-à-vis du cleva. Une telle interprétation, appliquée à $\breve{s} a l$, me semble assez difficile dans le contexte de la Tabula, où l'on parle d'une incision d'un texte officiel.

Par contre, il ne me semble point impossible que $\check{s} a / e l$ - exprime une confirmation, une ratification ou une approbation (cfr. Latin ratus, sancire), de façon que la phrase masan tiurunias šlace vacal tmial dans Pyrgi B puisse signifier: «le masan tiurunias a ratifié l'offrande du temple» («masan sanxit templi donum»). On peut très bien s'imaginer que Thefarie Veliunas ait fait construire le temple, l'ait dédié à base d'une promesse quelconque à Uni/Astarte et que quelque temps après la ratification de cette donation ait eu lieu.

Et le texte de la tablette de Cortona nous dit alors que «le šians a incisé ici sur la tablette le texte d'accord avec les frères Cušu et Petru». Il me semble donc tout à fait acceptable que l'on dise du texte original qu'il se trouve dans la maison

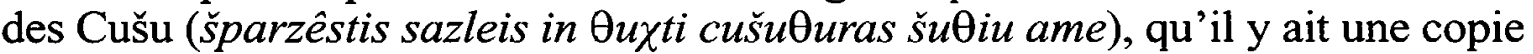
chez Petru (tal šu $\theta$ ivenas ratm $\theta u \chi \chi$ cesu tltel têi) et que seulement après on mentionne le fait que le texte a été incisé sur la tablette de bronze par un fonctionnaire quelconque et avec l'accord officiel des deux parties (šians šparzête $\theta$ ui šalt zic fratuce cušu $\theta$ uras larišališvla pêtrusc šcêvas). Même si normalement le locatif avec postposition $-t / \theta$ s'utilise dans la fonction locativale et non instrumentale, il ne me semble pas impossible que šal-t puisse quand même être interprété comme «'accord avec / avec la ratification de».

Les autres textes avec des formes dérivées de šal- ne nous apprennent pas grand chose. Il y a par exemple šelašva dan Vt 4.2 dont on n'ose pas affirmer qu'il s'agirait d'un pluriel inanimé d'une forme * šelaš qui nous présente le morphème agentif $-\check{s}^{(66)}$. Sur le plan du contenu il faut admettre qu'on ne sait quasiment rien. Puis il y a dans le Liber Linteus trois fois la forme šal (LL 6.1 (contexte très

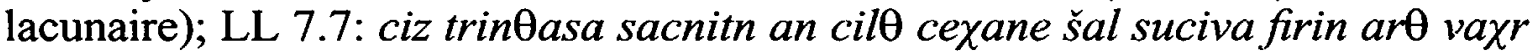

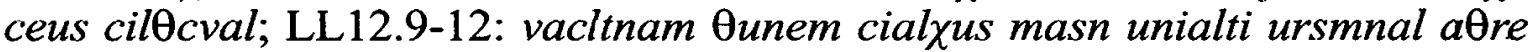
acil an sacnicn cilӨ cexa šal cus cluce). Quoiqu'il soit évident que ces textes ne peuvent rien prouver en ce qui concerne $\check{s} a l$, il faut remarquer quand même quelques parallélismes entre LL 7.7 et 12.9: il y a la séquence sacnitn/cn an

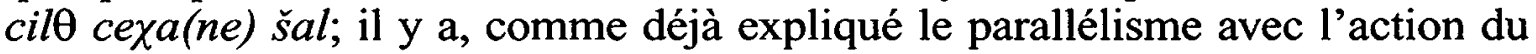

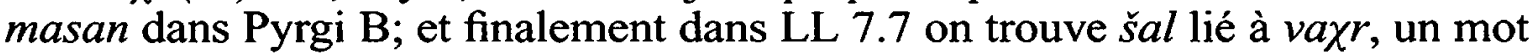

(64) Pfiffig, Uni-Hera-Astarte, 1965, p.38; Olzscha, dans Glotta, 44, 1967, p.101

(65) Appunti, p.71-72

(66) Cfr Wylin, dans $A G I, 89$ (2004), 1, p.111-127. 
qui pourrait signifier «contrat» ou «promesse» dans le Cippus Perusinus ${ }^{(6 i)}$. Ce qui prouve de nouveau une interprétation possible de $\check{s}$ al comme «confirmation, ratification».

(67) Cfr Facchetti, Frammenti, p.11. 